\title{
Bronchopulmonary dysplasia: effect of altitude correction and role for the Neonatal Research Network Prediction Algorithm
}

\author{
Kristina Gulliver $^{1} \cdot$ Bradley A. Yoder ${ }^{1}$
}

Received: 19 December 2017 / Revised: 14 March 2018 / Accepted: 16 March 2018 / Published online: 9 May 2018

(c) Nature America, Inc., part of Springer Nature 2018

\begin{abstract}
Objective To determine the effect of altitude correction on bronchopulmonary dysplasia (BPD) rates and to assess validity of the NICHD "Neonatal BPD Outcome Estimator" for predicting BPD with and without altitude correction.

Subjects Retrospective analysis included neonates born <30 weeks gestational age (GA) between 2010 and 2016. "Effective" $\mathrm{FiO}_{2}$ requirements were determined at 36 weeks corrected GA. Altitude correction performed via ratio of barometric pressure (BP) in our unit to sea level BP. Probability of death and/or moderate-to-severe BPD was calculated using the NICHD BPD Outcome Estimator.

Results Five hundred and sixty-one infants were included. Rate of moderate-to-severe BPD decreased from 71 to $40 \%$ following altitude correction. Receiver-operating characteristic curves indicated high predictability of BPD Outcome Estimator for altitude-corrected moderate-to-severe BPD diagnosis.

Conclusion Correction for altitude reduced moderate-to-severe BPD rate by almost $50 \%$, to a rate consistent with recent published values. NICHD BPD Outcome Estimator is a valid tool for predicting the risk of moderate-to-severe BPD following altitude correction.
\end{abstract}

\section{Introduction}

For infants born at $<32$ weeks gestation, bronchopulmonary dysplasia (BPD) is commonly defined by the need for supplemental oxygen at 36 weeks corrected gestational age (CGA) [1]. Recent reports suggest that there may be an increased BPD risk for preterm infants cared for at elevated altitudes [2,3]. It is unclear if the reported increased rate of BPD at higher altitudes is due to neonatal intensive care unit (NICU) management, or is related to the lower level of absolute oxygen pressure $\left(\mathrm{pO}_{2}\right)$ contributing to lower pulse oximetry saturations $\left(\mathrm{SpO}_{2}\right)$ [4-6]. The NICHD Neonatal Research Network recently developed a model for predicting BPD at various postnatal ages for neonates 23-30 weeks estimated GA with birth weight $401-1250 \mathrm{~g}$ [7]. The cohort used for developing the model was almost exclusively managed at low altitude NICU's. Our objectives

Kristina Gulliver

Kristina.gulliver@hsc.utah.edu

1 Division of Neonatology, University of Utah, Salt Lake City, UT, USA for this study were twofold: (1) to determine the effect of altitude correction for $\mathrm{FiO}_{2}$ on BPD rates, and (2) to assess the validity of the "Neonatal BPD Outcome Estimator" for predicting BPD in our high-risk preterm infants with and without altitude correction.

\section{Methods}

Following waiver of consent approval from our institutional review board, we performed a retrospective analysis from a prospectively collected neonatal database. Neonates born at $<30$ weeks estimated GA and managed in the University of Utah NICU from January 2010 through December 2016 were included in the study. Infants born with known fetal anomalies were excluded. Data included maternal and neonatal demographics, aspects of respiratory support, and neonatal outcomes up to the time of discharge from the NICU. Specific to respiratory support, we use highfrequency oscillatory ventilation as the initial mode of mechanical ventilation in premature infants. Conventional mechanical ventilation is subsequently employed at the attending physician's discretion using a volume-targeted approach. The mode of support and $\mathrm{FiO}_{2}$ were recorded at 
hours of life 168 (day 7) and 336 (day 14), 30 days after birth, and 36 weeks CGA. Per our unit guidelines, for infants on low flow nasal cannula oxygen, we performed a room air challenge at 36 weeks corrected age or prior to discharge, whichever occurred first, with $\mathrm{SpO}_{2}$ targeted to be at or above $92 \%$.

We defined BPD using the 2001 NICHD Consensus Conference criteria [1], with modification as follows: (1) no $\mathrm{BPD}$, in room air at 28 days postnatal age (PNA), and 36 weeks CGA; (2) mild BPD, on supplemental $\mathrm{O}_{2}$ by any source at 28 days PNA, and in room air at 36 weeks CGA; (3) moderate BPD, on supplemental $\mathrm{O}_{2}$ by any source at 28 days PNA, and on nasal cannula $\mathrm{O}_{2}$ with "effective $\mathrm{FiO}_{2} " 0.30$ at 36 weeks CGA; and (4) severe BPD, on supplemental $\mathrm{O}_{2}$ by any source at 28 days PNA, and either "effective $\mathrm{FiO}_{2}$ " >0.30 OR any positive pressure mode OR high flow nasal cannula $>1 \mathrm{~L} / \mathrm{min}$ at 36 weeks CGA. For infants on any form of nasal cannula $\mathrm{O}_{2}$, we estimated the "effective" $\mathrm{FiO}_{2}$ as follows to define the severity of PBD at 36 weeks. For infants receiving nasal cannula oxygen, we estimated the "effective" $\mathrm{FiO}_{2}$ using the equation described by Benaron and Benitz [8] via an online calculator (http:// pub.emmes.com/study/rop/stop-js.html). Inputs for the equation include current weight, cannula flow rate in liters per minute, and $\mathrm{FiO}_{2}$ in the delivered gas. Subsequently, we adjusted the diagnosis of moderate or severe BPD based on altitude correction for delivered effective $\mathrm{FiO}_{2}$ as follows. We determined the equivalent fraction of inspired oxygen at altitude relative to sea level using the ratio of barometric pressure (BP) equation: $\mathrm{FiO}_{2 \mathrm{sl}}=\mathrm{BP}_{\text {alt }} \times \mathrm{FiO}_{2 \mathrm{alt}} \times\left(100 / \mathrm{BP}_{\mathrm{sl}}\right)$. $\mathrm{BP}$ at sea level (sl) was assumed as $760 \mathrm{~mm} \mathrm{Hg}$. The BP in the NICU at the University of Utah (alt) at a measured altitude of $5000 \mathrm{ft}$ is typically around $640 \mathrm{~mm} \mathrm{Hg}$. Thus, in our NICU, an $\mathrm{FiO}_{2}$ of 0.209 (room air) is equivalent to an $\mathrm{FiO}_{2}$ of 0.177 at sea level, while an $\mathrm{FiO}_{2}$ of 0.248 in our unit is equivalent to an $\mathrm{FiO}_{2}$ of 0.209 at sea level.

We estimated the risk of moderate-to-severe BPD/death using the NICHD Neonatal Research Network BPD Outcome Estimator. The model includes the following parameters: estimated gestation (completed weeks) at birth, birth weight, sex, race or ethnicity, postnatal day, mode of respiratory support, and $\mathrm{FiO}_{2}$ [7]. Using the model's website (https://neonatal.rti.org) we calculated the sum probability of moderate BPD, severe BPD, or death at 7 and 14 days PNA. Due to the prediction model limitations for birth weight, we used the maximum allowable weight of $1249 \mathrm{~g}$ for neonates born $>1250 \mathrm{~g}$.

\section{Data analysis}

All statistical analyses were performed using SPSS (version 19 , IBM, Armonk, NY, USA). $\chi^{2}$ test was used for comparisons of uncorrected and altitude-corrected rates of BPD.
Table 1 Demographic characteristics of the study population $(n=622)$

\begin{tabular}{|c|c|}
\hline Gestational age (weeks) ${ }^{\mathrm{a}}$ & $27.0(1.9)$ \\
\hline Birth weight $(g)^{\mathrm{a}}$ & $963(301)$ \\
\hline Weight at 36 weeks CGA $(\mathrm{g})^{\mathrm{a}}$ & $2194(380)$ \\
\hline Male gender, $n(\%)$ & $337(54)$ \\
\hline Cesarean section, $n(\%)$ & $425(68)$ \\
\hline Inborn, $n(\%)$ & $560(90)$ \\
\hline 1-min Apgar ${ }^{\mathrm{b}}$ & $4(2-7)$ \\
\hline 5-min Apgar ${ }^{\mathrm{b}}$ & $7(6-8)$ \\
\hline Received surfactant, $n(\%)$ & $500(80)$ \\
\hline Received any betamethasone, $n(\%)$ & $569(92)$ \\
\hline Intubated, $n(\%)$ & $536(86)$ \\
\hline Age at intubation $(\min )^{b}$ & $6(3-12.5)$ \\
\hline Days on ventilator ${ }^{\mathrm{b}}$ & $4(0.1-30)$ \\
\hline $\mathrm{FiO}_{2}$ at 7 days $\mathrm{PNA}^{\mathrm{a}}$ & $0.30(0.11)$ \\
\hline $\mathrm{FiO}_{2}$ at 14 days $\mathrm{PNA}^{\mathrm{a}}$ & $0.33(0.14)$ \\
\hline $\mathrm{FiO}_{2}$ at 36 weeks $\mathrm{CGA}^{\mathrm{a}}$ & $0.31(0.17)$ \\
\hline Discharged home on supplemental $\mathrm{O}_{2}, n(\%)$ & $282 / 592(48)$ \\
\hline Maternal age (years) ${ }^{\mathrm{a}}$ & $28.1(6.4)$ \\
\hline \multicolumn{2}{|l|}{ Maternal race, $n(\%)$} \\
\hline Caucasian & $400(64)$ \\
\hline Black & $19(3)$ \\
\hline Hispanic & $145(23)$ \\
\hline Other & $58(9)$ \\
\hline
\end{tabular}

A one-sample $z$ test was used to compare BPD rates to the NRN BPD prediction score at 14 days PNA. Two-sided $P$ values $<0.05$ were considered statistically significant. No adjustments were made for multiple comparisons. Receiveroperating characteristics were determined using the dichotomous outcome of death or moderate-to-severe BPD vs. survival with no or mild BPD.

\section{RESULTS}

Between 1 January 2010 and 31 December 2016 we managed 656 infants born at $<30$ weeks estimated gestational age (EGA) in the University of Utah NICU. We excluded 34 for fetal anomaly. Of the remaining 622, 561 (90.2\%) neonates survived to 36 weeks CGA and were assessed for BPD. Demographic characteristics of the study population are shown in Table 1. Table 2 indicates the mode of respiratory support at 7 and 14 days PNA.

Of the 561 infants that survived to 36 weeks CGA, 400 (71\%) met the NICHD criteria for moderate-to-severe BPD based on their need for supplemental oxygen. As shown in 
Table 2 Mode of respiratory support at 7 and 14 days PNA

\begin{tabular}{lll}
\hline & $\begin{array}{l}7 \text { days PNA, } \\
n=599\end{array}$ & $\begin{array}{l}14 \text { days PNA, } \\
n=583\end{array}$ \\
\hline Room air & $52(9)$ & $51(9)$ \\
Nasal cannula & $124(21)$ & $145(25)$ \\
Nasal high flow therapy & $55(9)$ & $83(14)$ \\
CPAP/NIPPV & $126(21)$ & $91(16)$ \\
Conventional ventilator & $99(17)$ & $82(14)$ \\
High-frequency ventilator & $122(20)$ & $114(20)$ \\
\hline
\end{tabular}

Data are given as $n(\%)$

$N I P P V$ non-invasive positive pressure ventilation, $P N A$ postnatal age

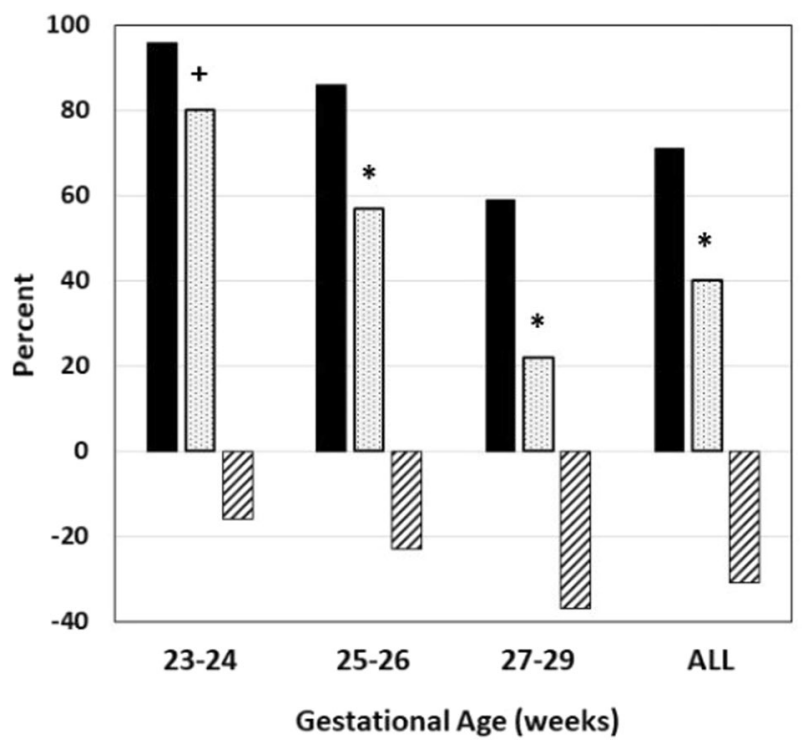

Fig. 1 Rate of uncorrected moderate-to-severe BPD at 36 weeks gestation (black bars) by gestational age. Following altitude correction (speckled bar), the rate of moderate-to-severe BPD at 36 weeks significantly decreased for each gestational age subgroup, and for all infants $<30$ weeks gestation $\left({ }^{+} P=0.003,{ }^{*} P<0.001\right)$. The percent decrease at each gestational age and overall are shown by the dashed bars

Fig. 1, the rate varied by GA, with $81 / 84(96 \%)$ at 23-24 weeks EGA, 124/144 (86\%) at 25-26 weeks EGA, and 195/333 (59\%) at 27-29 weeks EGA. The rate of moderate-to-severe BPD or death was not statistically different from the rate of moderate-to-severe BPD alone for all GAs.

Following correction of altitude, 223/561 (40\%) continued to meet the moderate-to-severe BPD criteria. Rates of moderate-to-severe BPD were significantly lower after altitude correction for all GAs and decreased with increasing GA, ranging from $67 / 84(80 \%)$ at $23-24$ weeks EGA to $74 / 333(22 \%)$ at $27-29$ weeks EGA. (Fig. 1) Thus, $44 \%$ (n, $177)$ of infants initially classified as moderate-to-severe BPD did not meet the diagnostic criteria following altitude adjustment. Among this group, 77 infants were reclassified

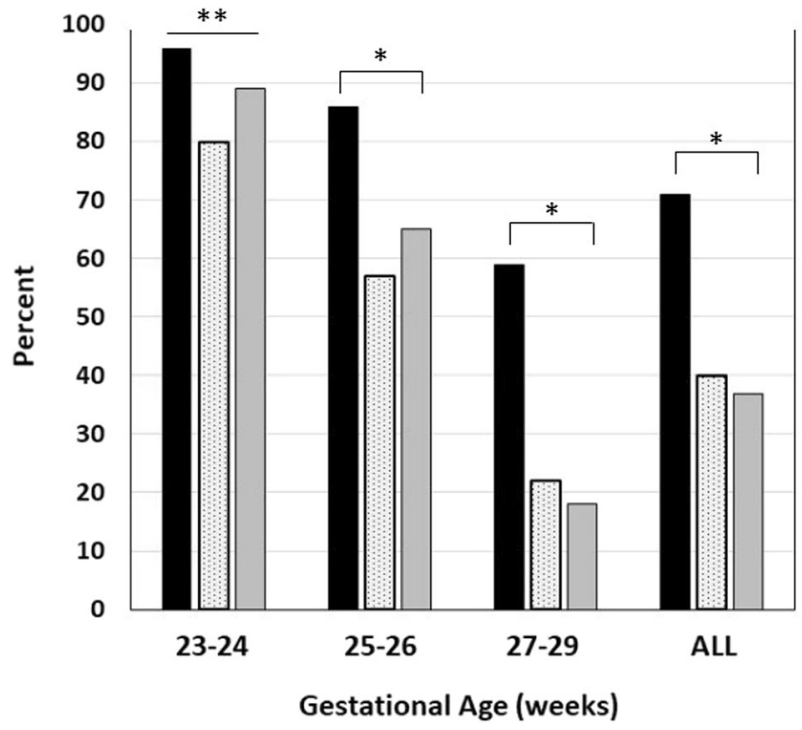

Fig. 2 Median predicted value for moderate-to-severe BPD using the Neonatal Research Network BPD Outcome Estimator (gray bar) compared to actual rates for moderate-to-severe BPD by gestational age subgroups before (black bar) and after (speckled bar) altitude correction. The NRN BPD Estimator more closely mirrors altitudecorrected rates for all comparisons except the 23-24 weeks subgroup. $* * P<0.05$ compared to uncorrected and altitude-corrected BPD rates by one-sample $z$ test. $* P<0.05$ compared to uncorrected BPD rate by one-sample $z$ test

as having no BPD, while 100 were reclassified from moderate-to-severe BPD to mild BPD. The relative change in corrected BPD rates increased with increasing GA as shown in Fig. 1.

Fig. 2, contrasts the corrected and uncorrected rates for moderate-to-severe $\mathrm{BPD} /$ death to the median predicted probability of moderate-to-severe BPD/death for each GA subgroup at 14 days PNA, as calculated from the NICHD Neonatal BPD Outcome Estimator. The median probability derived from the BPD calculator was significantly lower than the uncorrected BPD for all infants and subgroups, but closely approximates the corrected BPD rate for infants $>24$ weeks GA and for the entire study population.

Fig. 3, shows receiver-operating characteristic curves indicating high predictability of the Neonatal BPD Outcome Estimator for altitude-corrected diagnosis of moderate-tosevere BPD/death at both 7 and 14 days with area under the curve values of 0.859 (95\% confidence interval (CI): 0.82-0.890) and $0.873 \quad(95 \%$ CI: $0.844-0.901)$, respectively.

\section{DISCUSSION}

In this study, we showed that altitude correction for $\mathrm{FiO}_{2}$ reduced the apparent rate of moderate-to-severe BPD for infants $<30$ weeks EGA by almost $50 \%$. Additionally, we 


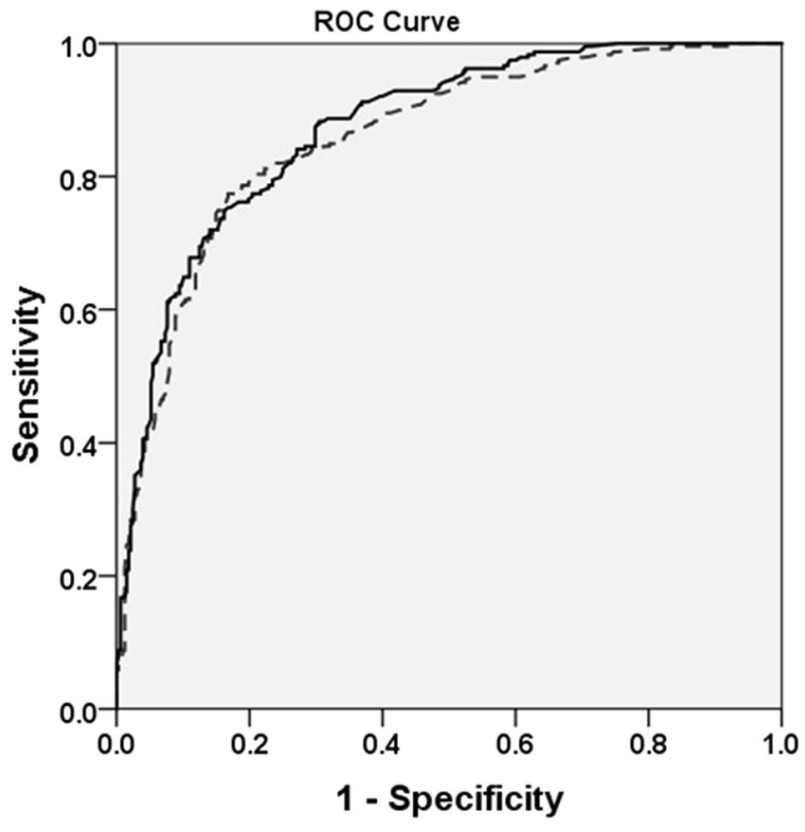

Fig. 3 Receiver-operating characteristic curves for the Neonatal Research Network BPD Outcome Estimator at 7 days (dashed line) and 14 days (solid line) for altitude-corrected moderate-to-severe BPD or death. Area under curve analysis: 7 days 0.859 (95\% CI: 0.82-0.89) and 14 days 0.873 (95\% CI: $0.844-0.901$ )

found that the NICHD BPD Outcome Estimator was a valid tool for predicting the risk of moderate-to-severe $\mathrm{BPD} /$ death in our NICU population following $\mathrm{FiO}_{2}$ correction for an altitude of $5000 \mathrm{ft}$.

The overall incidence of moderate-to-severe BPD at 36 weeks CGA in our population was $71 \%$ prior to altitude correction. This is in line with the uncorrected rate reported by Britton [9] in Denver, but less than the rates reported from studies outside the United States [3, 5]. However, the latter studies had older study groups and higher mortality rates which complicate comparisons. Our altitude-corrected rate for moderate-to-severe BPD of $40 \%$ is similar to the reported rate of $46 \%$ in a validation of the NICHD definition for moderate and severe BPD and of $43 \%$ in a recent report from the Prematurity and Respiratory Outcomes Program [10, 11].

One of the earliest studies to assess the role of altitude on outcomes among very low birth weight infants was by Paranka et al. [4]. In a multi-center cohort of over 5400 very low birth weight infants managed at varying altitudes, they found no effect on mortality or a variety of important neonatal morbidities, with the exception of oxygen use at 28 days. Specifically, they reported that over $65 \%$ of infants at $>4300 \mathrm{ft}$ were on supplemental oxygen at 28 days PNA compared to $48 \%$ cared for at lower altitudes. They did not report on the relative rates of BPD at 36 weeks or discharge to home on supplemental oxygen. Subsequently, Britton [9] reported on altitude correction for a small cohort of very low birth weight infants managed in Denver, Colorado at an altitude of $5280 \mathrm{ft}$. Using hood oxygen to adjust ambient $p \mathrm{O}_{2}$ for altitude, the diagnosis of BPD at 36 weeks CGA decreased from 72 to $27 \%$ [9]. A recent report from the Iberoamerican Neonatology network, comparing NICU's at the sea level to those over $7000 \mathrm{ft}$, also reported a fivefold decrease in the rate of moderate-to-severe BPD at 36 weeks following altitude correction [5]. Investigators from each of these studies posited that the predominant mechanism for difference in BPD rates at altitude was the difference in available $p \mathrm{O}_{2}$ at higher vs. lower altitude sites.

Other researchers have speculated that higher altitude alone may convey a significant risk for the development of BPD. Using data from the Canadian Neonatal Research Network, Lee et al. [2] reported a 9\% increase in odds of BPD or death with every $100 \mathrm{~m}(328 \mathrm{ft})$ increase in altitude. They speculated that the increased risk of BPD at higher altitude could be due to an exaggerated response to hypoxemia in preexisting vulnerable population. However, they had limited numbers of infants included at $>400 \mathrm{~m}$ $(1312 \mathrm{ft})$ and did not provide correction for lower $p \mathrm{O}_{2}$ as altitude increased. AlShehri [3] also reported increased rates for BPD at 36 weeks among preterm infants managed at high altitude vs. low altitude NICU's in Saudi Arabia. The author speculated early and prolonged exposure to higher $\mathrm{FiO}_{2}$ might contribute to an increased risk at altitude, but no attempt was made to correct for lower $p \mathrm{O}_{2}$ values at higher altitudes.

Our study is unique from other published studies in that we also assessed the probability for moderate-to-severe BPD, with and without altitude correction, using a wellconstructed predictive model $[7,12]$. We found that the NRN BPD Outcome Estimator applied at either 7 or 14 days postnatal age had excellent predictive ability for moderateto-severe BPD. Additionally, predictive values were more closely aligned with the diagnosis of BPD following altitude correction.

Strengths of this study include prospective data collection targeted toward the prediction of BPD using a validated model that is readily accessible and easy to use. Additionally, we assessed invasive and non-invasive respiratory parameters serially over several time points in an effort to assess consistency of the model. Finally, we targeted a highrisk cohort of preterm infants that may be eligible for comparative and preventative studies related to BPD. Study limitations include data from a single academic center.

In conclusion, BPD rates appear to be increased at higher altitude among infants born at $<30$ weeks gestation. Correction for altitude based on $\mathrm{BP}$, results in lower BPD rates that approximate those of infants managed closer to sea level. The Neonatal Research Network BPD Outcome Estimator is useful in predicting rates for moderate-tosevere BPD or death at higher altitudes. Future trials and 
definitions related to BPD should consider estimating effective $\mathrm{FiO}_{2}$ and altitude correction when comparing outcomes to lower altitude sites.

Author contributions $\mathrm{KG}$ and BAY contributed to the conception and design of the study, data collection and analysis, and manuscript preparation. Both authors approved the final manuscript as submitted and agree to be accountable for all aspects of the work.

\section{Compliance with ethical standards}

Conflict of interest The authors declare that they have no conflict of interest.

\section{References}

1. Jobe AH, Bancalari E. Bronchopulmonary dysplasia. Am J Respir Crit Care Med. 2001;163:1723-9.

2. Lee SK, Ye XY, Singhal N, De La Rue S, Lodha A, Shah PS, for the Canadian Neonatal Network. Higher altitude and risk of bronchopulmonary dysplasia among preterm infants. Am J Perinatol. 2013;30:601-6.

3. AlShehri MA. Are preterm infants at high altitude at greater risk for the development of bronchopulmonary dysplasia? J Trop Pediatr. 2014;60:68-73.

4. Paranka M, Brown M, Thomas P, Peabody J, Clark R. Are very low birth weight infants born at high altitude at greater risk for adverse outcomes? J Pediatr. 2001;139:669-72.

5. Fernández CL, Fajardo CA, Favareto MV, Hoyos A, Jijón-Letort FX, Carrera MS, for the Iberoamerican Society of Neonatology
Network. Oxygen dependency as equivalent to bronchopulmonary dysplasia at different altitudes in newborns $1500 \mathrm{~g}$ at birth from the SIBEN network. J Perinatol. 2014;34:538-42.

6. Bakr AF, Habib HS. Normal values of pulse oximetry in newborns at high altitude. J Trop Pediatr. 2005;51:170-3.

7. Laughon MM, Langer JC, Bose CL, Smith PB, Ambalavanan N, Kennedy KA, for the Eunice Kennedy Shriver National Institute of Child Health and Human Development Neonatal Research Network. Prediction of bronchopulmonary dysplasia by postnatal age in extremely premature infants. Am J Respir Crit Care Med. 2011;183:1715-22.

8. Benaron DA, Benitz WE. Maximizing the stability of oxygen delivered via nasal cannula. Arch Pediatr Adolesc Med. 1994;148:294-300.

9. Britton JR. Altitude, oxygen and the definition of bronchopulmonary dysplasia. J Perinatol. 2012;32:880-5.

10. Ehrenkranz RA, Walsh MC, Vohr BR, Jobe AH, Wright LL, Fanaroff AA, for the Eunice Kennedy Shriver National Institute of Child Health and Human Development Neonatal Research Network. Validation of the National Institutes of Health consensus definition of bronchopulmonary dysplasia. Pediatrics. 2005; 116:1353-60.

11. Keller RL, Feng R, DeMauro SB, Ferkol T, Hardie W, Rogers EE. et al., for the Prematurity and Respiratory Outcomes Program. Bronchopulmonary dysplasia and perinatal characteristics predict 1 -year respiratory outcomes in newborns born at extremely low gestational age: a prospective cohort study. J Pediatr. 2017; 187:89-97.

12. Onland W, Debray TP, Laughon MM, Miedema M, Cools F, Askie LM, et al. Clinical prediction models for bronchopulmonary dysplasia: a systematic review and external validation study. BMC Pediatr. 2013;13:207. 